\title{
Impact of the COVID-19 pandemic on Clinical Incidents and Complaints at a UK Teaching Hospital.
}

William Atiomo ${ }^{1,2}$, Peter Weir ${ }^{2}$ and Lucy Kean ${ }^{1}$.

1. Division of Family Health, Nottingham University Hospitals NHS Trust, Queens Medical Centre, Derby Road, Nottingham, NG7 2UH,

2. School of Medicine, University of Nottingham, B Floor, Medical School, Queens Medical Centre, Nottingham. NG7 2UH.

*Corresponding author: William.atiomo@nottingham.ac.uk 


\title{
Impact of the COVID-19 pandemic on Clinical Incidents and Complaints at A UK Teaching Hospital.
}

\author{
William Atiomo, Peter Weir and Lucy Kean.
}

\begin{abstract}
Background: To investigate any associations between new clinical policies implemented because of the COVID-19 pandemic and harm to patients.

Methods: Retrospective data collection of incidents and complaints reported through Datix ${ }^{\circledR}$, and the Patient Liaison Service respectively. The setting was the Family Health division in a University teaching hospital in the UK. Primary and secondary outcome measures included; Proportion of incidents reported on Datix from 23/3/20 to 25/5/20, compared to the period from $23 / 3 / 19$ to $29 / 5 / 19$. COVID-19 related incidents and complaints and association with newly published guidelines or pathways from 23/3/20 to 29/5/20.
\end{abstract}

Results: There was no significant difference in the proportion of overall patient activity resulting in incidents reported on Datix in 2020 (2.08\%) compared to 2019 (2.09\%), with 98\% resulting in no/low harm in 2020. Three incident categories had increases in relative proportions of incidents including terms "COVID" or "Corona" compared to incidents that did not; "Child death", "delay/failure to treatment and procedure" and "information governance". One of the child deaths was a miscarriage and we were unable to link the second child death to a change in clinical policy at this stage. We were only able to link 2 COVID-19 associated incidents with a pathway or procedural change (one to the Children's Emergency Department admission pathway and the second to the introduction of virtual antenatal clinics). Eighteen complaints related to COVID-19 were logged. However, at this stage, we are unable to link any of these to a published change in clinical policy.

\section{Conclusions:}

Practice in the division was overall deemed to be safe in the designated period, with only 2 COVID-19 related incidents clearly related to a change in pathways and procedures. Continued surveillance and improved metrics for monitoring the impact of changes to pathways and procedures should be sought with the sustained presence of COVID-19 in clinical areas.

Keywords: COVID, Corona, Incidents, Complaints, Obstetrics, Gynaecology, Paediatrics, Child, Quality, Safety. 


\title{
Impact of the COVID-19 pandemic on Clinical Incidents and Complaints within the Family Health Division at A UK Teaching Hospital.
}

\author{
William Atiomo, Peter Weir and Lucy Kean.
}

\section{BACKGROUND}

The Coronavirus disease 2019 (COVID-19), [1] pandemic resulted in an unprecedented change in global health care delivery. As at $7^{\text {th }}$ September 2020, 27,438,479 cases had been reported globally with unfortunately 895,440 deaths. The corresponding figures in the UK, were, 350,100 cases and 41,554 deaths, [2]. In response to the pandemic, in a televised address, the British prime minister, Boris Johnson announced a UK-wide partial lockdown, to contain the spread of the virus. The British public were instructed that they must stay at home, except for certain "very limited purposes" - shopping for basic necessities; for "one form of exercise a day"; for any medical need; and to travel to and from work when "absolutely necessary", [3].

Several measures were also rapidly introduced by hospitals in the United Kingdom to cope with the additional potential burden of the pandemic. National Health Service (NHS) England for example announced that all non-urgent treatment would be postponed from 15 April to free up to 30,000 beds with many hospitals introducing virtual consultations, [4] to reduce the number of face to face consultations to minimize the risks of transmitting the COVID-19 infection and ensure patient safety.

Locally, at our hospital, Nottingham University Hospital (NUH) NHS Trust in response to the COVID-19 pandemic a series of new and/or revised clinical pathways and procedures were rapidly implemented, the majority of which were still in effect as of August 2020. Prior to this study, there had however been no study investigating the impact of these changes to health service delivery on patient safety. This study therefore aimed to investigate any association between changes in health care delivery, introduced as a result of the COVID-19 and harm to patients within the Family Health (FH) division at NUH. Specifically, we were investigating whether any new pathways or processes were associated with increased harm, as we prepared to restore our departmental services to treat an increased number of patients as the COVID-19 lockdown restrictions were gradually lifted. This was to inform how possible shortcomings could be addressed to ensure safe practice in the continued presence of COVID-19. The focus on the FH division which covers Obstetrics, Gynaecology, Paediatrics (Children's Hospital), and Sexual Health was because the authors were in the senior management team of the division and felt it was very important to inform their decision making on objective data on patient safety. The exercise was also an opportunity to provide a framework to inform safe restoration of clinical services in collaboration with our health care system partners. 


\section{METHODS}

This was a retrospective study carried out in June 2020. Ethics committee approval was not thought to be required for the study because it was a desk-based service review, that did not involve any patient contact. The Medical Research Council (MRC) Regulatory Support Centre / the UK NHS Health Research Authority (HRA) online decision support tool (http://www.hra-decisiontools.org.uk/research/), also, did not class the study as research.

Data collection, covering the time period 23/3/20 - 29/5/20 was collected. The date range was selected as it represented the first 9 weeks following the UK wide lockdown, whilst preceding the restoration and recovery of clinical pathways in the NUH FH division.

Incidents and complaints in the division were reported by people in a range of roles, including medical and non-medical staff, patients, carers, parents, or guardians. Data on all incidents logged on the Datix ${ }^{\circledR}$ software, and complaints registered with the Patient Advise and Liaison Service (PALS) were obtained. In addition, details of all procedural changes and revised protocols in this time period (23/03/20-29/05/20) were obtained. Data collection was carried out by the FH clinical effectiveness team, the NUH PALS team and staff in the data support unit. All retrieved data was transferred to a Microsoft Excel ${ }^{\circledR}$ spreadsheet for analysis by one of the authors. The data was analyzed as follows: A Datix and free text search for "covid" or "corona" was performed by two authors (WA and PW). Following this, any incidents identified as relating to "covid" or "corona" were read and linked to the title of a published new clinical policy in response to the COVID-19 pandemic by one author (WA). Data from 23/3/20 29/5/20, was compared with data from 23/3/19 - 29/5/19 (similar period one year earlier). Data was then summarized as proportions. The significance of statistical comparisons between categorical variables was calculated using the chi-squared test. A p value of less than 0.05 was considered significant for this purpose. Statistical analyses were done using a publicly available statistical package available at

http://www.obg.cuhk.edu.hk/ResearchSupport/StatTools/Unpaired2Props_Pgm.php [last accessed 7 September 2020].

\section{Patient and Public Involvement.}

No patients or members of the public were involved in this study.

\section{RESULTS}

\section{Patient Activity Data.}

There were 7,138 inpatient episodes and 25,099 outpatient attendances in the NUH FH division from $23 / 3 / 20$ to $25 / 9 / 20$ just after the COVID-19, lockdown. A total patient activity count of 32,237 . The numbers for $23 / 3 / 19$ to $29 / 5 / 19$, before the COVID-19 lockdown, were 9,411 inpatient episodes and 30,027. A total activity count of 39,438. This represented a $24 \%, 16 \%$ and $18 \%$ drop in inpatient episodes, outpatient attendances and total patient activity count respectively, in the NUH FH division. 


\section{Incident reporting rates.}

There was no statistically significant difference in the proportion of incidents reported on Datix from $23 / 3 / 20$ to $29 / 5 / 20$, compared to the period from 23/3/19 to 29/5/19. Six hundred and seventy-two (672) (2.08\% of overall patient activity) incidents were reported in 2020 compared to 826 (2.09\% of overall patient activity) incidents reported in 2019. Of these, one serious untoward incident $(0.0031 \%$ of the overall patient activity) was reported in 2020 and one serious untoward incident $(0.0025 \%$ of the overall patient activity) was reported in 2019 . The serious incident in 2020 involved a baby born in poor condition following a via forceps delivery, whilst the serious incident in 2019 involved delayed recognition of jaundice in 7-day old baby.

Although the serious untoward incident in 2020 following the COVID-19 lockdown was still being investigated at the time of data analysis, there was no obvious indication to suggest that it arose because of a change in health care delivery pathway or procedure by the NUH FH division, introduced in response COVID-19 pandemic.

Most (656 out of 672 (98\%)) of the incidents reported after the COVID-19 pandemic in 2020 resulted in no or low harm. The corresponding figures in 2019 were 821 out of 826 (99\%). There was no statistically significant difference found in the proportion of incidents classed as resulting in low or no harm in 2020 compared with 2019. There was however a significant increase in the number of incidents reported as resulting in moderate harm in 2020 compared to $2019(12(1.79 \%)$ vs $4(0.48 \%)), p<0.05$.

\section{COVID-19 related incidents and association with newly published guidelines or pathways in NUH FH Division $(23 / 3 / 20$ to $29 / 5 / 20$.}

Twenty-eight (28) patients with diagnosed COVID-19 infection were admitted to the NUH FH division in the above period but no deaths were recorded. Fifty-six (56) new clinical policies were created across the division during the study period. Of these, $28(50 \%)$ were in the children and young people's (paediatrics) services, $13(23 \%)$ in maternity, $9(16 \%)$ in gynaecology, and $6(11 \%)$ in sexual health (Table 1).

Table 1. Procedural changes and revised policies in NUH FH division; 23.03.20 to 29/05/20.

\begin{tabular}{|l|l|}
\hline \multicolumn{2}{|c|}{ Procedural Documents and revised processes - Covid 19: Family Health Division } \\
\hline Speciality & revised practice due to Covid-19 \\
\hline Children \& Young People & Community Paediatricians' deployment areas, April 2020 \\
\hline Children \& Young People & Community Paediatrics Handbook \\
\hline Children \& Young People & COVID-19: GP Referrals: A guide for paediatric consultants - 18th March 2020 \\
\hline Children \& Young People & Master COVID-19 Rota 23.03.20 \\
\hline Children \& Young People & Paediatric PPE Guidance For use in Nottingham Children's Hospital \\
\hline Children \& Young People & Outpatient Timetable \\
\hline
\end{tabular}


Children \& Young People Children \& Young People Children \& Young People Children \& Young People Children \& Young People Children \& Young People Children \& Young People Children \& Young People Children \& Young People Children \& Young People Children \& Young People Children \& Young People Children \& Young People Children \& Young People Children \& Young People Children \& Young People Children \& Young People Children \& Young People Children \& Young People Children \& Young People Children \& Young People Children \& Young People Gynaecology Gynaecology Gynaecology

Gynaecology

Gynaecology

Gynaecology

Gynaecology

Gynaecology

Gynaecology

Sexual Health Services

Sexual Health Services

Sexual Health Services

Sexual Health Services

Sexual Health Services

Sexual Health Services

Maternity

Maternity

Maternity

Maternity

Maternity

Maternity

Maternity

Maternity
Outpatient Update Covid-19 Flow chart

Paediatric Time Critical Surgery Prioritisation Principles

PRM Team Response -Covid 19 respiratory team plan

Safeguarding Children and Young People

TRENT CLEFT NETWORK - Planning document for COVID-19.

Resus and PCCU (email)

Manager's guide to supporting students on extended clinical placement

Covid-19 Education and Training Process

Final Paper - PCCU (email)

Final Paper - PCCU BCH (email)

PICU Escalation for receiving adult patients draft V1 (email)

PICU Escalation for receiving adult patients V1 (email)

Urgent - drug stock HIGH DEPENDENCY (E40) (email)

Requirements to open Paediatric HDU beds on ward E40 (Annex Bay) : COVID 19 surge plan. March 2020

Covid Planning PCCU flowchart

NUH PCCU Collaboration with $\mathrm{BCH}$ v2

Drug Stock for HIGH DEPENDENCY E40 (email)

Children's Hospital Skills Refresher Sessions March 2020

ACS Covid Programme

Training Process for Covid-19 Emergency register Staff

Working in Teams

FFP3 Fit Test Drop in Sessions

2 week wait Gynaecological

COVID pathway for Emergency Gynaecology \& early pregnancy

Daily SITREP and DOWNREP communication within FHD

Management of a Confirmed case of Covid-19

Management of a suspected case of Covid=19

A23 inpatient/outpatient standard operating procedure for contraception -Covid-19

A23 PO Clinicians guide final

Standard letters for deferment of benign-urogynaecology clinic appointments

Urology Pathway During Covid Crisis

ISHS telephone triage - COVID Emergency

ISHS telephone triage - COVID Emergency

Urology Pathway During Covid Crisis 01.04.2020

NUH ISHS expedited partner treatment - COVID Emergency

NUH ISHS expedited partner treatment - COVID Emergency

Covid 19 Patient Check List

Advanced Maternity care guideline

Algorithm Selected antenatal -BP self-monitoring

Selected* antenatal hypertension protocol

Hospital staff - quick guide to changes for postnatal discharges

IOL Risk assessment

Self-monitoring blood pressure in pregnancy and post-natal - Quick Guide

Coronavirus (COVID-19) Postnatal Care Pathway

Standard Operating Procedure

Performing NIPE's during COVID-19 pandemic

Covid 19 Maternity SOP 


\begin{tabular}{|l|l|} 
Maternity & Obstetric ultrasound guidance document in response to Covid 19 Final \\
\hline Maternity & CV19 Looking After Yourself \\
\hline Maternity & CV19 Planning Your Birth \\
\hline Maternity & Revised Reporting Requirements (for information only) \\
\hline Maternity & OOH calls process map 1.4.20 Version 1.0 Final \\
\hline
\end{tabular}

Of the 672 incidents reported, 61 (9\% of reported incidents) included the words "COVID" or "Coronavirus". COVID-19 related incidents were further classified by degree of resulting harm, and 52 were classed as resulting in no harm to the patient, (85\% of COVID-19 related incidents) 6 low harm (10\% of COVID-19 related incidents), 2 moderate harm (3\% of COVID19 related incidents) and in 1 case the degree of harm (2\% of COVID-19 related incidents) was not stated in Datix, which was the case of a child death (a young boy (in the age range 10-15 years old) who died from septic shock). Overall, 95 of all the 61 COVID-19 related incidents from resulted in no or low harm (Figure 1). Three of the 61 COVID-19 related incidents involved patients infected directly with the virus.

Three incident categories (table 2) had statistically significant increases in relative proportions of incidents including terms "COVID" or "Corona" compared to the set of incidents that did not include these terms; "Child death" ( $\Delta$ (difference) $3 \%, \mathrm{p}=0,0472$ ), "delay/failure to treatment and procedure" $(\Delta 10.3 \%, \mathrm{p}=0,0123)$ and "information governance" $(\Delta 8.4 \%$, $\mathrm{p}=0,003$ ). Table 3 provides more details of these incidents. With respect to the child deaths, there were two incidents reported after the COVID-19 pandemic between 23/3/20 - 29/5/20. We were unable to clearly link either of these 2 child deaths with a pathway or procedural change in FH. One child death was an inevitable miscarriage and the second child death was a young boy (in the age range 10-15 years old) who died from septic shock. Three of the four incidents of aggression, violence, or harassment were consequences of Trust wide changes to the policy relating to relatives visiting patients. At the stage of data analysis, without further detailed root cause analyses, we were only able to link 2 Covid-19 associated incidents with a pathway or procedural change in FH (one to the Children's emergency department (ED) admission pathway and the second to the introduction of virtual antenatal clinics). 
Table 2. Incident categories with statistically significant increases between Covid-19 related and non-Covid-19 related incidents in the period (23/3/20 to 29/5/20).

\begin{tabular}{|c|c|c|c|c|c|c|}
\hline Category & $\begin{array}{l}\text { Number of } \\
\text { COVID-19 } \\
\text { related } \\
\text { incidents } 2020 \\
\text { ( } \mathrm{n}=61 \text { ) }\end{array}$ & $\begin{array}{l}\text { Number of } \\
\text { non-COVID- } \\
19 \text { related } \\
\text { incidents } \\
(\mathrm{n}=611)\end{array}$ & $\begin{array}{l}\text { Percentage } \\
\text { of (COVID- } \\
19 \text { related } \\
\text { incidents } \\
2020 \text { ) }\end{array}$ & $\begin{array}{l}\text { Percentage of } \\
\text { non-COVID-19 } \\
\text { related incidents. }\end{array}$ & $\begin{array}{l}\text { Percentage } \\
\text { difference. COVID } \\
19 \text { versus non- } \\
\text { COVID } 19 \text { related } \\
\text { incidents. }\end{array}$ & $\begin{array}{l}p \\
\text { values. }\end{array}$ \\
\hline Child Death & 2 & 2 & $3.3 \%$ & $0,3 \%$ & $3,0 \%$ & 0,0472 \\
\hline $\begin{array}{l}\text { Delay / failure } \\
\text { to treatment } \\
\text { or procedure }\end{array}$ & 11 & 47 & $18.0 \%$ & $7,7 \%$ & $10,7 \%$ & 0,0123 \\
\hline $\begin{array}{l}\text { Information } \\
\text { Governance }\end{array}$ & 7 & 19 & $11.5 \%$ & $3,1 \%$ & $8,6 \%$ & 0,0039 \\
\hline
\end{tabular}

Table 3. Association between clinical incident and pathway or procedural change in NUH FH division.

\begin{tabular}{|c|c|c|c|}
\hline Category & Degree of Harm & $\begin{array}{l}\text { Association with a } \\
\text { pathway or procedural } \\
\text { change specific to the } \\
\text { NUH FH Division. }\end{array}$ & Source \\
\hline Child Death * & None & No & \\
\hline Child Death ** & Not stated & No & \\
\hline $\begin{array}{l}\text { Delay / failure to treatment } \\
\text { or procedure }\end{array}$ & Low & No & \\
\hline $\begin{array}{l}\text { Delay / failure to treatment } \\
\text { or procedure }\end{array}$ & Moderate & No & \\
\hline $\begin{array}{l}\text { Delay / failure to treatment } \\
\text { or procedure } * * *\end{array}$ & None & Yes & $\begin{array}{l}\text { Children's ED } \\
\text { admission pathway. }\end{array}$ \\
\hline $\begin{array}{l}\text { Delay / failure to treatment } \\
\text { or procedure }\end{array}$ & None & No & \\
\hline $\begin{array}{l}\text { Delay / failure to treatment } \\
\text { or procedure }\end{array}$ & None & No & \\
\hline $\begin{array}{l}\text { Delay / failure to treatment } \\
\text { or procedure }\end{array}$ & None & No & \\
\hline $\begin{array}{l}\text { Delay / failure to treatment } \\
\text { or procedure }\end{array}$ & None & No & \\
\hline $\begin{array}{l}\text { Delay / failure to treatment } \\
\text { or procedure }\end{array}$ & None & No & \\
\hline $\begin{array}{l}\text { Delay / failure to treatment } \\
\text { or procedure }\end{array}$ & None & No & \\
\hline $\begin{array}{l}\text { Delay / failure to treatment } \\
\text { or procedure }\end{array}$ & None & No & \\
\hline
\end{tabular}




\begin{tabular}{|c|c|c|c|}
\hline $\begin{array}{l}\text { Delay / failure to treatment } \\
\text { or procedure }\end{array}$ & None & No & \\
\hline Information Governance & Moderate & No & \\
\hline $\begin{array}{l}\text { Information Governance } \\
* * * *\end{array}$ & None & Yes & $\begin{array}{l}\text { Virtual antenatal } \\
\text { clinics }\end{array}$ \\
\hline Information Governance & None & No & \\
\hline Information Governance & None & No & \\
\hline Information Governance & None & No & \\
\hline Information Governance & None & No & \\
\hline Information Governance & None & No & \\
\hline \multicolumn{4}{|c|}{$\begin{array}{l}\text { * Inevitable miscarriage. Unable to prevent outcome. Issue with timely documentation because medical } \\
\text { staff present at time of delivery on shift had been drafted from other specialties to support A23 } \\
\text { (gynaecology emergency ward) during COVID-19 and therefore processes unfamiliar to them. }\end{array}$} \\
\hline \multicolumn{4}{|c|}{ ** a young boy (in the age range 10-15 years old) who died from septic shock. } \\
\hline \multicolumn{4}{|c|}{$\begin{array}{l}\text { *** Admission pathway for children with suspected covid to go to emergency department (ED) first. } \\
\text { Patient arrived in ED as a GP expect with a history of a high temperature at home. Due to temperature } \\
\text { being a COVID symptom, ED rang ward to say that the patient could be admitted straight to D33. } \\
\text { Difficulty contacting medical registrar and SHO. Well over an hour before seen by a doctor. }\end{array}$} \\
\hline \multicolumn{4}{|c|}{$\begin{array}{l}\text { **** "As part of our new processes in response to COVID-19, a summary of the antenatal assessment } \\
\text { journal from the entry onto Maternity Medway recorded after the telephone consultation is sent to the } \\
\text { woman, GP and community midwife along with a cover letter, to substitute for documenting in the part } 1 \\
\text { maternity record. The consultant doing the consultation previews the summary to check that this is } \\
\text { appropriate. On previewing this woman's record sensitive information about domestic abuse was visible } \\
\text { on the record". }\end{array}$} \\
\hline
\end{tabular}

\section{COVID-19 related complaints and association with newly published guidelines or pathways in NUH FH Division $(23 / 3 / 20$ to $29 / 5 / 20$.}

In the same time period (23/3/20 to 29/5/20) 18 complaints related to COVID-19 were logged through PALS. However, at the stage of data analysis, without formal investigation of the complaints, we were unable to patently connect/link any of these to a published pathway or procedural change. The complaints were spread across four categories (Table 4). Six complaints regarding clinical treatment, five complaints regarding patient safety, four complaints regarding communication and three complaints regarding appointments.

Table 4. Association between complaints and pathway or procedural change in NUH FH division.

\begin{tabular}{|l|l|l|}
\hline Specialty & Subjects & $\begin{array}{l}\text { Was the complaint obviously } \\
\text { associated with a published } \\
\text { pathway or procedural change } \\
\text { in the Family health Division. }\end{array}$ \\
\hline
\end{tabular}




\begin{tabular}{|l|l|l|} 
Obstetrics & M - Safety & No \\
\hline Gynaecology & D - Communication & No \\
\hline Children and Young People & M - Safety & No \\
\hline Obstetrics & C - Clinical Treatment & No \\
\hline Obstetrics & C - Clinical Treatment & Uncertain \\
\hline Obstetrics & D - Communication & Uncertain \\
\hline Obstetrics & C - Clinical Treatment & Uncertain \\
\hline Obstetrics & M - Safety & No \\
\hline Fertility Clinic (Andrology) & B - Appointments & No \\
\hline Children and Young People & D - Communication & No \\
\hline Obstetrics & M - Safety & No \\
\hline Obstetrics & M - Safety & No \\
\hline Children and Young People & D - Communication & No \\
\hline Obstetrics & C - Clinical Treatment & Uncertain \\
\hline Obstetrics & B - Appointments & Uncertain \\
\hline Gynaecology & B - Appointments & Uncertain \\
\hline Children and Young People & C - Clinical Treatment & No \\
\hline Obstetrics & C - Clinical Treatment & No \\
\hline
\end{tabular}

\section{DISCUSSION}

We were unable to find any similarly published studies in our literature review in a literature search of the PubMed database using the following search terms; "Covid" AND "complaints" and "Covid" AND "incidents"). However, one pre-print (pre-peer review) study identified on a search on the "Google" search engine, [5] from Imperial College Healthcare NHS Trust, (a group of 5 hospitals located in central London), found that error reporting measured from Datix, as we did in our study, significantly reduced. The authors found that in the 8 weeks following the first Covid-19 patient arriving at the trust, the number of weekly error reports consistently fell below the 52-week mean and that on 6 of the 8 weeks, the rate was more than 3 standard deviations below the weekly mean. Our study also found a reduction of the numbers of incidents reported, however, when corrected for the of overall patient activity, the proportions of incidents reported before and after the COVID-19 lockdown were not statistically significant.

We also found a study from Nepal published in the Lancet Global Health, [6] which found that institutional births were reduced by about $50 \%$ with an increased risk of preterm births, still births and neonatal mortality during lockdown. We however did not set out to measure these indices in our study, although the drop in births mirrors the $24 \%$ drop in patient activity we observed in our study.

The proportion of incidents classed as resulting in low or no harm in our study (98\%) was also similar to latest the national figure from the national patient safety incident reports (NaPSIR) for England (97\%), [7] which provides some reassurance that despite the outbreak of the COVID-19 pandemic, health care delivery in the NUH FH division remained safe. This is also 
reflected in the fact that the proportion of incident reported did not decrease. The fact that the proportion of incidents reported did not decrease with the additional demands imposed by the pandemic such that the Parliamentary and Health Service Ombudsman paused their work on existing NHS complaints and acceptance of new health complaints from 26 March 2020 to 30 June 2020, [8] was also reassuring as it suggested continued vigilance for patient safety in the NUH FH division.

In the designated study period, the service provided by the NUH FH division was overall safe, as the majority of activities (97.9\%) were not associated with any registered incidents. Furthermore, of the incidents registered, $85 \%$ resulted in no harm to patients. Our interest primarily lay in the association, or lack thereof between incidents and changes to our procedures and pathways. We were only able to definitely link 2 incidents to a published pathway or procedural change in FH (one to the Children's ED admission pathway and the second to the introduction of virtual antenatal clinics). From this we deduce that measures taken to avoid COVID-19 transmission and sequelae have at large not been to the detriment of patient safety in the division.

The study is limited by the relatively short period of data collection, prompted by the rapidly changing clinical picture during the early pandemic. Ideally, the data used to inform decisions around restoration and recovery plans would have been gathered over a longer period to improve the quality of decision making. There are however no obvious indications that any newly implemented policies require immediate reversal. Therefore, a process of continuous monitoring and reassessment of data as we gradually transition into "regular" clinical practice appears to be safe, and will help improve further, evidence-based decision making. A set of agreed upon metrics need to be established to efficiently surveil outcomes. These metrics need to be tailored to the altered style of clinical practice during the pandemic, for example virtual clinics. Decisions regarding clinical policies should ideally consider qualitative data and expert opinions. This would hopefully address some of the rigidity in our categorical outcome measures, and highlight less quantifiable aspects of clinical safety during the pandemic. Methods that may be beneficial in improving our understanding include questionnaire surveys of patient experience (e.g. on virtual consultations), focus groups of staff, and other metrics required to undertake a full Quality Impact Assessment (QIA) or Equality and Quality Impact Assessment, [9,10]. Which includes addressing: Impact on duty of quality (CQC/constitutional standards), patient safety, clinical outcomes, patient experience, staff experience and equality and diversity

For future reassessment and a potential complete QIA, a number of pitfalls should be addressed. Although the similar proportion of incidents reported before and after the pandemic does not suggest this, it is may be that the increased pressures and demands of the pandemic reduced incident reporting, so we may not know the full impact yet. This may be addressed by updating the methods and metrics for monitoring incidents and complaints. Another limitation of this study, was that certain other metrics e.g. missed cancer diagnosis and morbidity and mortality in the community, was not captured by the methods and scope of this study. These 
issues require vigilance in further follow-up studies, and the impact of which will unfortunately only become apparent belatedly.

\section{Conclusions.}

Despite the COVID-19 pandemic, the NUH FH division continued to provide a safe service overall, as there was no difference in the proportion of incidents reported on Datix just after the COVID-19 lockdown in 2020, compared a similar period in 2019. There was also no statistically significant difference found in the proportion of incidents classed as resulting in serious incidents or low or no harm. At the stage, of submission of this article, we were able to link only two Covid-19 associated incidents with a new policy in response to the COVID19 pandemic, in the NUH FH division; one to the Children's ED admission pathway and the second to the introduction of virtual antenatal clinics.

Based on this initial assessment we consider it safe to extend the pathways and procedures introduced in response to COVID-19, without risk of significant detriment to patient safety/experience in FH. Whether it be in the midst of a pandemic or not, a serious incident in a 2- month period is still a figure we should strive to reduce. By initiating a longer-term followup process investigating our procedures and pathways, with improved metrics and data collection, we hope to minimize the additional impact caused by the COVID-19 pandemic in Family Health at NUH. We also hope that this study provides a useful framework for conducting similar studies in other settings to determine the national/international impact of the COVID-19 pandemic on overall patient safety.

\section{List of abbreviations}

QIA Quality Impact Assessment

COVID-19 Coronavirus disease 2019

NHS National Health Service

NUH Nottingham University Hospital

FH Family Health

PALS Patient Advise and Liaison Service

\section{Declarations}

\section{Ethics approval and consent to participate}

Ethics committee approval was not thought to be required for the study because it was a deskbased service review, that did not involve any patient contact. The Medical Research Council (MRC) Regulatory Support Centre/ the UK NHS Health Research Authority (HRA) online 
decision support tool (http://www.hra-decisiontools.org.uk/research/), also, did not class the study as research.

\section{Consent for publication}

Not applicable

\section{Availability of data and materials}

The datasets used and/or analysed during the current study are available from the corresponding author on reasonable request.

\section{Competing interests}

The authors declare that they have no competing interests

\section{Funding}

No funding supported this study.

\section{Author Contributions}

William Atiomo; Substantial contributions to the conception or design of the work; acquisition, analysis and interpretation of data for the work; AND Drafting the work and revising it critically for important intellectual content; AND Final approval of the version to be published; AND Agreement to be accountable for all aspects of the work in ensuring that questions related to the accuracy or integrity of any part of the work are appropriately investigated and resolved.

Peter Weir: Substantial contributions to the analysis and interpretation of data for the work; AND Drafting the work and revising it critically for important intellectual content; AND Final approval of the version to be published; AND Agreement to be accountable for all aspects of the work in ensuring that questions related to the accuracy or integrity of any part of the work are appropriately investigated and resolved.

Lucy Kean: Substantial contributions to the conception of the work; AND revising it critically for important intellectual content; AND Final approval of the version to be published; AND Agreement to be accountable for all aspects of the work in ensuring that questions related to the accuracy or integrity of any part of the work are appropriately investigated and resolved.

\section{Acknowledgements:}

We would like to thank Enzani Nyatoro, Angela Philips, Alison Pancoe and Halima Dagmia for their help with data collection for this study. 


\section{REFERENCES:}

1) Zhu N, Zhang D, Wang W, et al. A Novel Coronavirus from Patients with Pneumonia in China, 2019. N Engl J Med. 2020;382(8):727-33.

2) Coronavirus Reported Cases and Deaths by Country, Territory, or Conveyance. https://www.worldometers.info/coronavirus/ (accessed $31^{\text {st }}$ August 2020).

3) Wikipedia contributors, 'Timeline of the COVID-19 pandemic in England', Wikipedia, The Free Encyclopedia, 7 September 2020, 11:49 UTC, $<$ https://en.wikipedia.org/w/index.php?title=Timeline_of_the_COVID19_pandemic_in_England\&oldid=977185365> [accessed 7 September 2020]

4) Gilbert AW, Billany JCT, Adam R, et al Rapid implementation of virtual clinics due to COVID-19: report and early evaluation of a quality improvement initiative $B M J$ Open Quality 2020;9:e000985. doi: 10.1136/bmjoq-2020-000985.

5) Denning M, Goh ET, Scott A, et al. What has been the impact of Covid-19 on Safety Culture? A case study from a large metropolitan teaching hospital. medRxiv; 2020. DOI: $10.1101 / 2020.06 .15 .20129080$.

6) Kc A, Gurung R, Kinney MV, et al. Effect of the COVID-19 pandemic response on intrapartum care, stillbirth, and neonatal mortality outcomes in Nepal: a prospective observational study [published online ahead of print, 2020 Aug 10]. Lancet Glob Health. 2020;S2214-109X(20)30345-4.

7) NHS England and NHS Improvement. NRLS national patient safety incident reports: commentary. March 2020.

https://improvement.nhs.uk/documents/6571/NAPSIR_commentary_March_2020.pdf [accessed 7 September 2020].

8) Parliamentary and Health Service Ombudsman. Coronavirus: Information for complaint handling teams. https://www.ombudsman.org.uk/organisations-weinvestigate/coronavirus-information-complaint-handling-teams [accessed 7 September 2020].

9) National Quality Board. 2012. HOW TO: Quality Impact Assess Provider Cost Improvement Plans.

https://assets.publishing.service.gov.uk/government/uploads/system/uploads/attac hment_data/file/212819/How-to-Quality-Impact-Assess-Provider-CostImprovement-Plans-.pdf [accessed 7 September 2020].

10) NHS Improvement; 2018. Developing Workforce Safeguards. https://improvement.nhs.uk/documents/3320/Developing_workforce_safeguards.p df. [accessed 7 September 2020]. 
FIGURE LEGENDS:

COVID-19 related incidents in FH 23/3/20 - 29/5/20

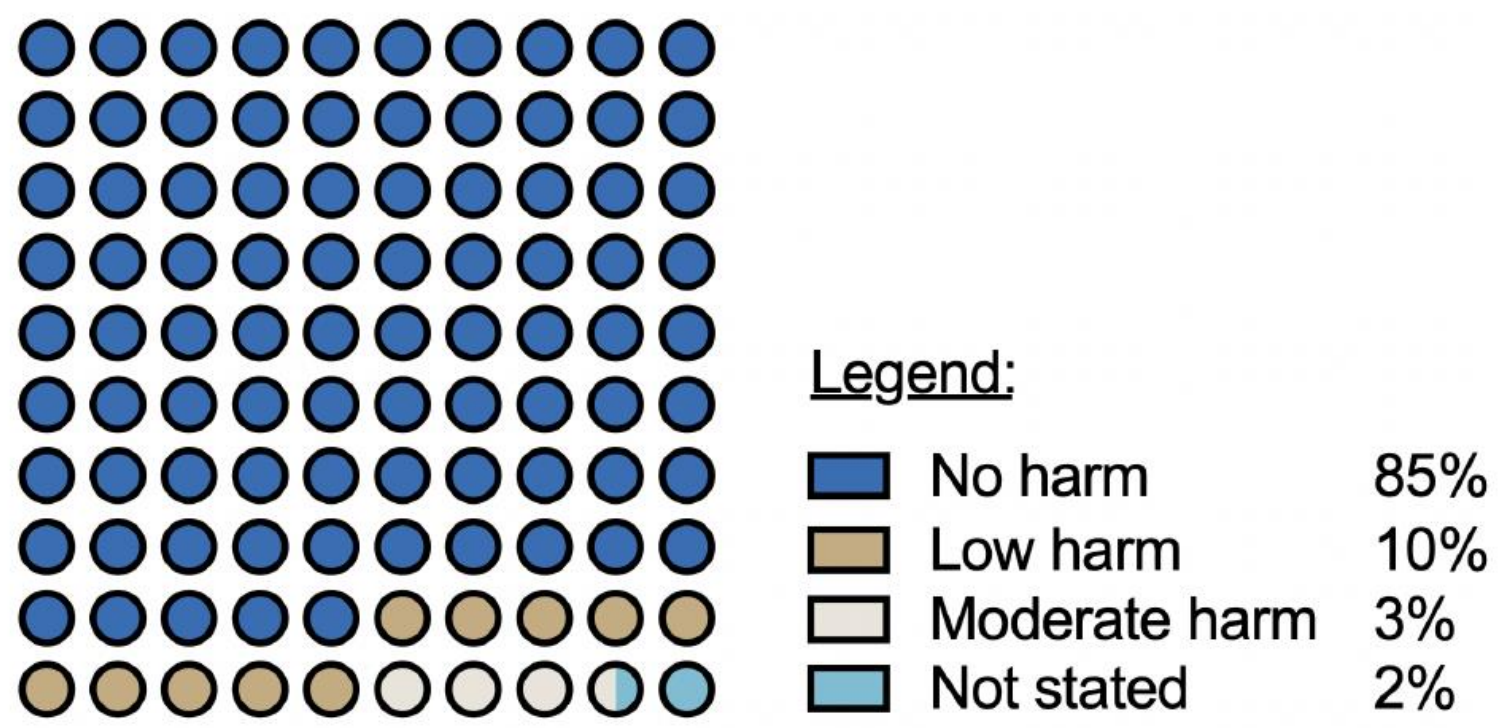

\section{Total Covid incidents $=61$}

Figure 1: The degree of harm resulting from incidents deemed to be Covid-19 related in the period $(23 / 3 / 20$ to $29 / 5 / 20)$ : No harm $(n=52)$, low harm $(n=6)$, moderate harm $(n=2)$, not stated $(\mathrm{n}=1)$. 